\title{
RÉGLAGE AUTOMATIQUE DES GHAMBRES DE WILSON
}

\author{
Par R. RICHARD-FOY. \\ Ingénieur de l'Aéronautique. \\ Laboratoire de Physique de l'École Polytechnique.
}

\begin{abstract}
Sommaire. - Il faut modifier le réglage d'une chambre de Wilson quand la température $t$ varie, en augmentant le taux de détente quand $t$ augmente. Il suffit, pour rendre ce réglage automatique, d'une modification simple du régulateur de pression employé au Laboratoire des radiations de Berkeley.
\end{abstract}

Une des difficultés de l'emploi pendant un temps long d'une chambre de Wilson est sa sensibilité aux variations de température. On peut y remédier partiellement par une climatisation de la salle d'expériences, mais à haute altitude en montagne ou en avion ce procédé est difficile à mettre en œuvre correctement.

Nous avons donc envisagé d'étudier un appareil qui fasse varier automatiquement le taux de détente en fonction de la température. Si nous utilisons une chambre dont la détente est produite par le relâchement d'une pression d'air comprimé, la position avant du piston étant déterminée par l'équilibre de la pression sur les deux faces du piston et la position arrière étant fixe, cet appareil peut être constitué par le régulateur de pression d'air comprimé convenablement modifié.

Nous allons d'abord exposer le principe du régulateur utilisé au laboratoire de radiation de Berkeley ( $\left.{ }^{1}\right)$ et montrer comment on peut le modifier pour obtenir le résultat que nous cherchons.

A. Régulateur ordinaire d'air comprimé. Ce régulateur se compose des chambres $x, 2$ et 3 que l'on voit sur la figure I, sans la chambre O. La chambre 1 et la chambre 3 sont séparées par une membrane de caoutchouc sur laquelle est collée la pièce métallique A qui forme la chambre 2. La chambre 3 communique avec l'appareil consommateur d'air comprimé et la chambre 2 est reliée à la distribution d'air comprimé par un pointeau creux P. On voit que si la pression en 3 est plus faible qu'en 1 la membrane se déplace vers le haut et dégage le pointeau de son siège, permettant à l'air de passer de 2 en 3 ce qui rétablit l'équilibre.

$\mathrm{Si}$, par contre, la pression en 3 était plus forte qu'en 1 , rien ne viendrait modifier l'équilibre si l'appareil utilisateur ne comportait pas de fuites, pratiquement il en a toujours et l'équilibre s'établit grâce à elles. Ce régulateur ne fonctionne donc pas pour un débit nul, il exige de légères fuites. La chambre 1 se termine par un tombac dont le volume est réglable, ce qui permet de faire varier la presion $p$

() Je remercie W. B. Fretter de m'avoir indiqué ce principe. dans 1 et de régler ainsi avec précision la position avant du piston du Wilson. Ce tombac ne comporte pas la tuyauterie $L_{1}$ figurée sur la figure $\mathrm{r}$.

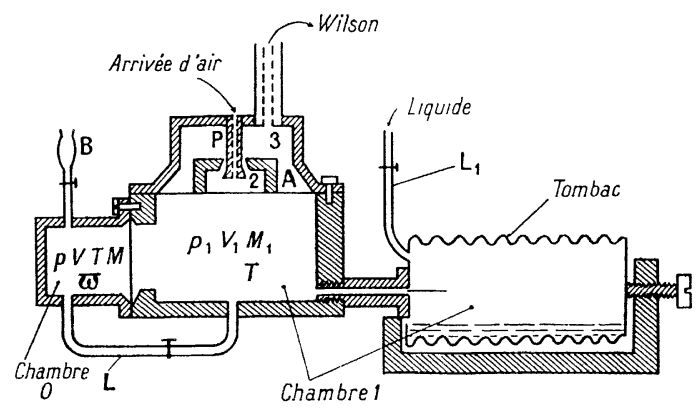

Fig. r. - Réglage automatique des chambres de Wilson.

La précision du réglage dépend du rapport du volume total $V_{1}$ de la chambre 1 à la surface $S$ de la membrane. La précision de la régulation dépend du rapport de la force $f$ nécessaire à assurer la fermeture du pointeau à la surface $S$ de la membrane d'une part, et d'autre part, du rapport de $V_{1}$ à $S$.

On a donc intérêt, pour avoir un appareil précis, à prendre

$$
\frac{F_{1}}{y} \text { arand et } f \text { prolit. }
$$

Pratiquement, on peut considérer que la pression en 3 reste toujours égale à celle en 1 en négligeant $\frac{j}{s}$ et en tous cas les variations de pression provoquées par une perturbation quelconque en 1 et 3 restent toujours égales.

B. Régulateur compensé. - Si la température $T$ varie de +$\lrcorner T$, tous les volumes qui interviennent dans l'appareil restant fixés, les pressions varient également et la position avant du piston reste inchangée, elle a même tendance à reculer légèrement, car la pression de vapeur saturante augmente plus rapidement que celle du gaz sec; le taux de détente tend donc plutôt à diminuer, alors que l'expérience montre que le bon réglage est obtenu par une augmentation de ce taux . 
Pour remédier à ce défaut nous proposons d'adjoindre au régulateur précèdent une chambre 0 séparée de la chambre I par une membrane élastique et après avoir établi l'équilibre de pression entre 0 et 1 au moyen de la tuyauterie $L$ d'y introduire un liquide très volatil de tension de vapeur $\varpi$. Nous allons calculer la variation $\frac{\mathrm{d} p_{1}}{\mathrm{~d} T}$.

La chambre 0 est caractérisée par son volume $V$; la pression $p$; la masse $M$ de gaz sec; la température $T$ et la pression $\varpi$ de vapeur. La chambre 1 par $V_{1}, p_{1}, M_{1}$ et $T_{1}$.

Nous avons

$$
\begin{gathered}
p V=K M T \\
p_{1} V_{1}=K M_{1} T \\
p_{1}=p+\pi \quad \text { (équilibre de la membrane } .
\end{gathered}
$$

d'où

$$
\begin{gathered}
\frac{\mathrm{d} p}{p}+\frac{\mathrm{d} V}{r}=\frac{\mathrm{d} t}{T}, \quad \frac{\mathrm{d} p_{1}}{p_{1}}+\frac{\mathrm{d} V_{1}}{V_{1}}=\frac{\mathrm{d} t}{T}, \\
\mathrm{~d} p_{1}=\mathrm{d} p+\mathrm{d} \pi .
\end{gathered}
$$

On élimine $\mathrm{d} p, \mathrm{~d} V, \mathrm{~d} V_{1}$ et l'on fait en première approximation $p=p_{1}$, d'où

$$
\frac{\mathrm{d} p_{1}}{\mathrm{~d} T}=\frac{p_{1}}{T}+\frac{V}{V+V_{1}} \frac{\mathrm{d} \pi}{\mathrm{d} T} .
$$

Revenons maintenant à la chambre de Wilson de volume $V_{4}$, pression $p_{4}$, température $T$ en position comprimée et de volume fixe $V_{5}$ en position détendue.

Nous avons $V_{5}=(\mathrm{I}+\varepsilon) V_{4}$ par définition et $p_{4} \# p_{1}$ comme nous l'avons vu avec $\mathrm{d} p_{4}=\mathrm{d} p_{1}$. Soit $p_{4}^{\prime}$ la pression du gaz dans la chambre et $\varpi_{4}$ la pression de vapeur saturante, on a

$$
s_{4}=p_{4}^{\prime}+\omega_{4}, \quad p_{4}^{\prime} V_{4}=K M_{4} T .
$$

Une variation de température $T$ donne une variation de volume $V$ telle que

$$
\begin{gathered}
\frac{\mathrm{d} p_{4}^{\prime}}{p_{4}^{\prime}}+\frac{\mathrm{d} V_{4}}{V_{4}}=\frac{\mathrm{d} T}{T} \quad \text { et } \quad \mathrm{d} p_{4}=\mathrm{d} p_{4}^{\prime}+\mathrm{d} \varpi_{4} \quad\left(1^{b i s}\right) \\
\frac{\mathrm{d} V_{4}}{V_{4}}=\frac{\mathrm{d} t}{T}-\frac{\mathrm{d} p_{4}}{p_{4}^{\prime}}+\frac{\mathrm{d} \varpi_{4}}{p_{4}^{\prime}} \# \frac{\mathrm{d} T}{T}=\frac{\mathrm{d} p_{1}}{p_{1}}+\frac{\mathrm{d} \varpi_{4}}{p_{1}} .
\end{gathered}
$$

Or

$\frac{\mathrm{d} V_{4}}{V_{4}}=\frac{-\mathrm{d} \varepsilon}{\mathrm{I}+\varepsilon}, \quad$ donc $\quad \frac{\mathrm{d} \varepsilon}{\mathrm{I}+\varepsilon}=\frac{-\mathrm{d} T}{T}+\frac{\mathrm{d} p_{1}}{p_{1}}-\frac{\mathrm{d} \varpi_{1}}{p_{1}}$.

Reportons. la valeur de $\frac{\mathrm{d} p_{1}}{p_{1}}$ tirée de (1), nous avons

Donc

$$
\frac{\mathrm{d} \varepsilon}{\mathrm{I}+\varepsilon}=\frac{\mathrm{I}}{p_{1}}\left(\mathrm{~d} \pi \frac{V}{V+V_{1}}-\mathrm{d} \pi_{4}\right) \text {. }
$$

$$
\frac{\mathrm{d} \varepsilon}{\mathrm{d} T}=\frac{\mathrm{I}+\varepsilon}{p}\left(\frac{\mathrm{d} \omega}{\mathrm{d} T} \frac{V}{V+V_{\cdot 1}}-\frac{\mathrm{d} \varpi_{4}}{\mathrm{~d} T}\right) .
$$

Le régulateur fait donc varier automatiquement le taux de détente. Si nous connaissons la quantité $\frac{\mathrm{d} \varepsilon}{\mathrm{d} T_{\exp }}$ qui correspond à une conservation du bon réglage, l'équation ci-dessus permet de calculer le rapport $\frac{V}{V+V_{1}}$ des chambres 0 et 1 du régulateur compensant automatiquement les variations de température. Pratiquement l'on construira un régulateur dont les volumes des chambres se rapprocheront des valeurs calculées et l'on fera un réglage espérimental en faisant varier le volume $V_{1}$. Pour cela il suffit d'adjoindre au tombac la tuyauterie $L_{1}$ par laquelle on versera un volume connu d'un liquide à faible tension de vapeur dans la cavité 1 . On pourra, par exemple, utiliser de l'huile pour basse pression.

D'autre part, en raison des forces parasites provoquées par l'élasticité des différentes membranes, il est préférable de déterminer directement la variation $\left(\frac{\mathrm{d} p_{1}}{\mathrm{~d} T}\right)_{\text {exp }}$ correspondant à un bon réglage au lieu de $\frac{\mathrm{d} \varepsilon}{\mathrm{d} T_{\text {cxp }}}$ et de calculer $\frac{V}{V+V_{1}^{\prime}}$ à partir de cette fonction.

Pour une chambre de Wilson fonctionnant à la pression ordinaire à l'eau et alcool, $\varepsilon$ est de l'ordre de $0, \mathbf{I}_{2}$, avec $\frac{\mathrm{d} \varepsilon}{\mathrm{d} T}$ de l'ordre de o,oor et $\frac{\mathrm{d} \varpi_{4}}{\mathrm{~d} T}$ de l'ordre de I,7 mm de $\mathrm{Hg}$. Nous avons donc en ordre de grandeur

$$
0,00 \mathrm{I} \sim \frac{\mathrm{I}, \mathrm{I}}{-60} \frac{\mathrm{d} \pi}{\mathrm{d} T}\left(\frac{V}{V+\Gamma_{1}} \quad 1.7\right)
$$

c'est-à-dire

$$
\frac{\mathrm{d} \varpi}{\mathrm{d} T} \frac{V}{V+V_{1}} \sim 0.7+1,-.
$$

Il faut donc choisir un liquide dont la pression de vapeur saturante ait une variation $\frac{\mathrm{d} w}{\mathrm{~d} T}=2,5 \mathrm{~mm} \mathrm{Hg}$ par degré. Ceci est parfaitement réalisable.

Conclusion. - Nous pensons donc que l'utilisation d'un régulateur de ce type peut permettre un réglage aútomatique de la détente dans certaines limites et donner plus de souplesse à l'emploi de la chambre de Wilson.

Bien entendu, cela ne résout qu'une partie des problèmes que posent les variations de température; la condensation sur les parois à l'intérieur de la chambre et la variation de la composition chimique de l'atmosphère au cours du temps doivent être combattus par d'autres moyens.

Manuscrit reçu le 8 juin 1948 . 